PROCEEDINGS OF THE

AMERICAN MATHEMATICAL SOCIETY

Volume 140, Number 7, July 2012, Pages 2347-2356

S 0002-9939(2011)11077-3

Article electronically published on October 26, 2011

\title{
CAN ONE DISTINGUISH QUANTUM TREES FROM THE BOUNDARY?
}

\author{
PAVEL KURASOV
}

(Communicated by Hart F. Smith)

\begin{abstract}
Schrödinger operators on metric trees are considered. It is proven that for certain matching conditions the Titchmarsh-Weyl matrix function does not determine the underlying metric tree; i.e. there exist quantum trees with equal Titchmarsh-Weyl functions. The constructed trees form one-parameter families of isospectral and isoscattering graphs.
\end{abstract}

\section{INTRODUCTION}

This article is devoted to quantum graphs, more precisely to Schrödinger operators on metric graphs. Every such operator is determined by the triple

- the metric graph $\Gamma$ leading to the Hilbert space $L_{2}(\Gamma)$,

- the real potential $q \in L_{2}(\Gamma)$ leading to the Schrödinger differential operator $-\frac{d^{2}}{d x^{2}}+q$ on the edges,

- the matching and boundary conditions at internal and boundary vertices respectively, establishing couplings between the edges and ensuring that the differential operator is selfadjoint.

Our aim here is to discuss the most general inverse problem, i.e. the problem to determine all members of the triple from a certain set of spectral data. This problem can naturally be divided into three subproblems to reconstruct each particular member of the triple. Many authors discussed these subproblems separately without paying much attention to the most general inverse problem. One of the first tasks is to choose an appropriate set of spectral data meeting both mathematical and physical (practical) requirements. On the one hand, this set should guarantee unique solvability of the inverse problem and should also be minimal in some sense. On the other hand, all quantities in the set should have clear physical interpretations and be easily measurable in an experiment without destroying the quantum graph. In the current article we restrict ourselves to boundary measurements. By a graph's boundary we understand all vertices with valency one.

It is not a priori clear which set of spectral data is the most appropriate. Of course this set depends on the particular inverse problem under investigation. Thus for zero potential and standard matching/boundary conditions, to reconstruct the

Received by the editors August 14, 2010 and, in revised form, February 15, 2011.

2010 Mathematics Subject Classification. Primary 34L25, 81U40; Secondary 35P25, 81V99.

The author was supported in part by Swedish Research Council grant No. 50092501. 
metric graph it is enough to know just the spectrum of the operator, provided the edge lengths are rationally independent [14, 19]. Without the latter unnatural requirement, the spectrum alone in general does not determine a unique metric graph, unless the set of admissible operators is reduced further [10. This leads to the important notion of isospectral graphs. Several classes of isospectral graphs both with standard and certain nonstandard matching/boundary conditions were constructed 3, 4, 22, 23. Moreover graphs with cycles may have eigenfunctions with supports separated from the boundary. The corresponding eigenvalues may not be seen in boundary observations. To recover the potential the knowledge of just one spectrum is not sufficient even in the case of a single edge (single interval) 21. Extending the set of spectral data by including the Titchmarsh-Weyl matrix function (TW-function; see definition below) associated with all boundary vertices, one may reconstruct not only the metric tree (without any restriction on the edge lengths) but the potential as well, provided the matching/boundary conditions are standard [12, 13, 5, 6, 8, 9, 1, 24, 25. If the metric tree is known, then the potential and the matching conditions are also determined by the TW-function 2] under certain natural restrictions on the matching conditions; see also [16, 15] for the special case of a star graph and 11, where the method of spectral mappings is applied to a certain limited class of matching conditions. One may expect that these spectral data, i.e. the TW-function, would allow one to solve even the most general inverse problem for trees. The main goal of this article is to present a counterexample showing that the $\mathrm{TW}$-function in general does not determine uniquely the metric tree (of course provided the set of admissible matching conditions or potentials is not reduced further). We discovered that even in the case of a "cross" graph, i.e., the metric graph formed by two intersecting intervals (see Figure 1 below), the metric graph may not be uniquely determined by its TW-function. This phenomenon occurs due to a very special form of matching conditions at the central vertex and appears to be "rare" in the sense that almost all quantum trees are probably uniquely determined by their TW-functions.

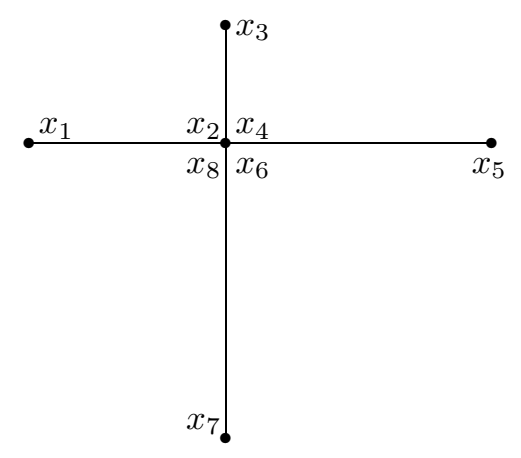

Figure 1. Graph $\Gamma$. Quantum cross. 
Constructing a one-parameter family of graphs with identical TW-functions we prove that quantum trees cannot always be distinguished using boundary measurements. The TW-function is in one-to-one correspondence with the dynamical response operator (the dynamical Dirichlet-to-Neumann map) and the scattering matrix and Lax-Phillips scattering operator for the noncompact graph obtained by attaching semi-infinite wires to all boundary vertices. Hence this matrix function encodes all information that can be obtained by boundary measurements. Extending the spectral data set further by including TW-functions associated with both boundary and internal vertices makes the inverse problem to recover the metric graph trivial, since the $\mathrm{TW}$-function determines the distances between all vertices. In the case of graphs with cycles, the class of graphs for which the inverse problem is uniquely solvable may be extended by introducing an additional magnetic potential and by considering spectral data depending on the magnetic fluxes [17, 18]. This method does not provide any new information in the case of trees, since the magnetic potential on trees can always be eliminated.

The counterexample presented here appears rather unexpected and is certainly important for the final solution of the inverse problem for quantum trees. In addition this construction leads to a new "continuum" family of isospectral graphs, which are also indistinguishable using boundary observations.

Families of quantum graphs with cycles having equal scattering matrices have already been constructed in [20, 7]. These graphs have different discrete spectra with eigenfunctions supported by the cycles. The corresponding eigenvalues are not determined by boundary measurements. The discussed quantum crosses have no cycles, and all their eigenfunctions are not zero near the boundary.

\section{The SELfadjoint OPERATOR}

Consider the "cross" graph $\Gamma$ formed by four intervals $\left[x_{2 j-1}, x_{2 j}\right], j=1, \ldots, 4$ joined together at the common internal vertex

$$
v_{5}=\left\{x_{2}, x_{4}, x_{6}, x_{8}\right\} .
$$

The boundary of this graph is formed by four vertices of valence 1:

$$
v_{j}=\left\{x_{2 j-1}\right\}, j=1,2,3,4 .
$$

This metric graph is determined by four positive real parameters, i.e., the lengths $l_{j}$ of the edges:

$$
l_{j}=x_{2 j}-x_{2 j-1} .
$$

Consider the Laplace operator in $L_{2}(\Gamma)$ defined on the domain of functions satisfying Dirichlet boundary conditions at the boundary vertices and certain special matching conditions at the central vertex, not the standard matching conditions as is often done 1 Let us denote by $L_{\sigma, \alpha}$ the second derivative operator $L=-\frac{d^{2}}{d x^{2}}$

\footnotetext{
${ }^{1} \mathrm{By}$ standard matching conditions we mean the conditions that the function is continuous and the sum of normal derivatives at the vertex is equal to zero.
} 
defined on the domain of functions $u$ from the Sobolev space $W_{2}^{2}\left(\Gamma \backslash\left\{x_{j}\right\}_{j=1}^{8}\right)$ satisfying the following matching conditions at the central vertex $v_{5}=\left\{x_{2}, x_{4}, x_{6}, x_{8}\right\}$ :

$$
\left(\begin{array}{cccc}
-1 & \alpha & 0 & \beta \\
\alpha & -1 & \sigma \beta & 0
\end{array}\right)\left(\begin{array}{l}
u\left(x_{2}\right) \\
u\left(x_{4}\right) \\
u\left(x_{6}\right) \\
u\left(x_{8}\right)
\end{array}\right)=\overrightarrow{0}, \quad\left(\begin{array}{cccc}
1 & \alpha & 0 & \beta \\
\alpha & 1 & \sigma \beta & 0
\end{array}\right)\left(\begin{array}{l}
u^{\prime}\left(x_{2}\right) \\
u^{\prime}\left(x_{4}\right) \\
u^{\prime}\left(x_{6}\right) \\
u^{\prime}\left(x_{8}\right)
\end{array}\right)=\overrightarrow{0}
$$

with $\alpha, \beta \in \mathbb{R}, \sigma= \pm 1$ subject to the constraint

$$
\alpha^{2}+\beta^{2}=1
$$

and Dirichlet conditions at the boundary vertices $v_{j}=x_{2 j-1}, j=1,2,3,4$,

$$
u\left(x_{1}\right)=u\left(x_{3}\right)=u\left(x_{5}\right)=u\left(x_{7}\right)=0 .
$$

The matching conditions (2.2) correspond to the energy independent vertex scattering matrix

$$
S=\left(\begin{array}{cccc}
0 & \alpha & 0 & \beta \\
\alpha & 0 & \sigma \beta & 0 \\
0 & \sigma \beta & 0 & -\sigma \alpha \\
\beta & 0 & -\sigma \alpha & 0
\end{array}\right)
$$

and can also be written in the standard form ([2]) as

$$
i(S-I)\left(\begin{array}{l}
u\left(x_{2}\right) \\
u\left(x_{4}\right) \\
u\left(x_{6}\right) \\
u\left(x_{8}\right)
\end{array}\right)=(S+I)\left(\begin{array}{l}
-u^{\prime}\left(x_{2}\right) \\
-u^{\prime}\left(x_{4}\right) \\
-u^{\prime}\left(x_{6}\right) \\
-u^{\prime}\left(x_{8}\right)
\end{array}\right) .
$$

These matching conditions have three important properties:

(1) the vertex scattering matrix is energy independent,

(2) the reflection coefficients are identically equal to zero,

(3) the transition coefficients to the opposite edges are identically equal to zero.

These properties of the matrix $S$ are crucial for the constructed counterexample. The operator $L_{\sigma, \alpha}$ is selfadjoint, and its spectrum is pure discrete satisfying the standard Weyl asymptotics.

\section{Calculation of the Titchmarsh-Weyl function}

The Titchmarsh-Weyl matrix function (TW-function) corresponding to the operator $L_{\sigma, \alpha}$ is defined as follows. Consider any function $u$ which is a solution to the eigenfunction equation

$$
-\frac{d^{2}}{d x^{2}} u=k^{2} u, \quad k^{2}=\lambda,
$$

on every edge for $\Im \lambda>0$ and satisfies the matching conditions (2.2). Every such function is uniquely determined by its values $u\left(x_{j}\right), j=1,3,5,7$ at the boundary vertices. (Otherwise the selfadjoint operator $L_{\sigma, \alpha}$ would have nonreal eigenvalues.) The $4 \times 4$ matrix $M(\lambda)$ connecting the vectors of boundary values and corresponding 
normal derivatives is called the Titchmarsh-Weyl function (TW-function):

$$
\left(\begin{array}{l}
u^{\prime}\left(x_{1}\right) \\
u^{\prime}\left(x_{3}\right) \\
u^{\prime}\left(x_{5}\right) \\
u^{\prime}\left(x_{7}\right)
\end{array}\right)=M(\lambda)\left(\begin{array}{l}
u\left(x_{1}\right) \\
u\left(x_{3}\right) \\
u\left(x_{5}\right) \\
u\left(x_{7}\right)
\end{array}\right) .
$$

Our aim is to calculate the matrix $M(\lambda)$ and to show that there exists a oneparameter family of graphs $\Gamma$ having identical TW-functions. Every solution to (3.1) can be written in the form

$$
u(x)=p_{j} \sin k\left(x-x_{2 j-1}\right)+q_{j} \cos k\left(x-x_{2 j-1}\right), \quad x \in\left(x_{2 j-1}, x_{2 j}\right), p_{j}, q_{j} \in \mathbb{C} .
$$

Substitution into the matching conditions (2.2) yields

$$
P \vec{p}+Q \vec{q}=0
$$

with

$$
P=\left(\begin{array}{cccc}
-\sin k l_{1} & \alpha \sin k l_{2} & 0 & \beta \sin k l_{4} \\
\alpha \sin k l_{1} & -\sin k l_{2} & \sigma \beta \sin k l_{3} & 0 \\
-\cos k l_{1} & -\alpha \cos k l_{2} & 0 & -\beta \cos k l_{4} \\
-\alpha \cos k l_{1} & -\cos k l_{2} & -\sigma \beta \cos k l_{3} & 0
\end{array}\right)
$$

and

$$
Q=\left(\begin{array}{cccc}
-\cos k l_{1} & \alpha \cos k l_{2} & 0 & \beta \cos k l_{4} \\
\alpha \cos k l_{1} & -\cos k l_{2} & \sigma \beta \cos k l_{3} & 0 \\
\sin k l_{1} & \alpha \sin k l_{2} & 0 & \beta \sin k l_{4} \\
\alpha \sin k l_{1} & \sin k l_{2} & \sigma \beta \sin k l_{3} & 0
\end{array}\right)
$$

Taking into account that $u\left(x_{2 j-1}\right)=q_{j}, u^{\prime}\left(x_{2 j-1}\right)=k p_{j}, j=1,2,3,4$ we conclude that the TW-function is given by

$$
M(\lambda)=-k P^{-1} Q
$$

The determinant of $P$ is

$$
\operatorname{det} P=\sigma \beta^{2}\left(\alpha^{2} \sin k\left(l_{1}-l_{3}\right) \sin k\left(l_{2}-l_{4}\right)-\sin k\left(l_{1}+l_{4}\right) \sin k\left(l_{2}+l_{3}\right)\right),
$$

which explains the following short notation:

$$
\left\{\begin{array}{ll}
c_{j}:=\cos k l_{j}, & s_{j}:=\sin k l_{j}, \\
c_{i \pm j}:=\cos k\left(l_{i} \pm l_{j}\right), & s_{i \pm j}:=\sin k\left(l_{i} \pm l_{j}\right),
\end{array} \quad i, j=1,2,3,4\right.
$$

Then the inverse matrix is

$$
\begin{aligned}
& P^{-1}=\frac{1}{\sigma \beta^{2}\left(\alpha^{2} s_{1-3} s_{2-4}-s_{1+4} s_{2+3}\right)} \times \\
& \left(\begin{array}{cccc}
\sigma \beta^{2} s_{2+3} c_{4} & \sigma \alpha \beta^{2} s_{2-4} c_{3} & \sigma \beta^{2} s_{2+3} s_{4} & \sigma \alpha \beta^{2} s_{2-4} s_{3} \\
\sigma \alpha \beta^{2} s_{1-3} c_{4} & \sigma \beta^{2} s_{1+4} c_{3} & \sigma \alpha \beta^{2} s_{1-3} s_{4} & \sigma \beta^{2} s_{1+4} s_{3} \\
-\alpha \beta s_{1+2} c_{4} & -\beta\left(\alpha^{2} s_{2-4^{c}} c_{1} s_{1+4} c_{2}\right) & -\alpha \beta s_{1+2} s_{4} & -\beta\left(\alpha^{2} s_{2-4^{s}}-s_{1+4} s_{2}\right) \\
-\sigma \beta\left(\alpha^{2} s_{1-3} c_{2}+s_{2+3} c_{1}\right) & -\sigma \alpha \beta s_{1+2} c_{3} & -\sigma \beta\left(\alpha^{2} s_{1-3} s_{2}-s_{2+3} s_{1}\right) & -\sigma \alpha \beta s_{1+2} s_{3}
\end{array}\right) .
\end{aligned}
$$


We obtain the following TW-function:

$(3.6)$

$$
\begin{aligned}
& M(\lambda)=
\end{aligned}
$$

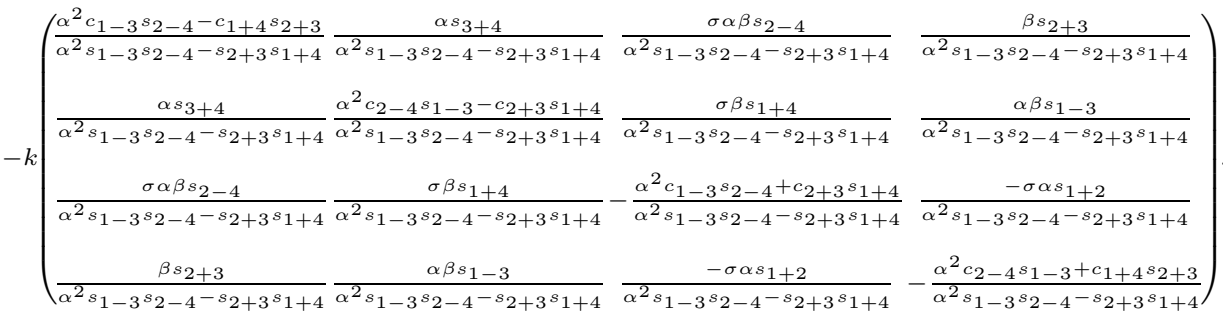

The matrix $M(\lambda)$ does not depend on all four length parameters $l_{j}, j=1,2,3,4$ determining the cross $\Gamma$. To see this, one may introduce the following three new length parameters:

$$
\begin{array}{ll}
\mathcal{L}=l_{1}+l_{2}+l_{3}+l_{4} & \text { - the total length of the graph, } \\
L_{1+2}=l_{1}+l_{2} & \text { - the distance between the vertices } v_{1} \text { and } v_{2}, \\
L_{1+4}=l_{1}+l_{4} & \text { - the distance between the vertices } v_{1} \text { and } v_{4} .
\end{array}
$$

It is easy to see that all other combinations of $l_{j}$ appearing in (3.6) can be expressed in terms of $\mathcal{L}, L_{1+2}, L_{1+4}$ as follows:

$$
\left\{\begin{array}{l}
l_{2}+l_{3}=\mathcal{L}-L_{1+4}, \\
l_{1}-l_{3}=L_{1+2}+L_{1+4}-\mathcal{L}, \\
l_{2}-l_{4}=L_{1+2}-L_{1+4} .
\end{array}\right.
$$

We have proven that the TW-functions for graphs with the edge lengths $l_{1}, l_{2}, l_{3}$, $l_{4}$ and $l_{1}+l, l_{2}-l, l_{3}+l, l_{4}-l$ are identical for any $0 \leq l<\min l_{j}$.

Lemma 3.1. The Laplace operators on cross graphs with the same distances between the neighbouring 2 boundary vertices have identical Titchmarsh-Weyl functions, provided the domain is defined by the matching conditions (2.2) at the central vertex and Dirichlet conditions on the boundary.

Among the four distances between neighbouring boundary vertices, just three distances are independent; the fourth distance and the total length of the graph can be calculated.

\section{Discussions And GENERALIZATIONS}

We shall now give an elementary explanation for the observed phenomenon. Consider the wave equation on $\Gamma$ determined by the operator $L_{\sigma, \alpha}$ :

$$
L_{\sigma, \alpha} u(x, t)=-\frac{\partial^{2}}{\partial t^{2}} u(x, t),
$$

subject to the boundary control

$$
u\left(x_{2 j-1}, t\right)=f_{j}(t), j=1,2,3,4 .
$$

For simplicity we assume that the function $u$ satisfies zero initial data and that the functions $f_{j}$ are smooth, $f_{j} \in C_{0}^{\infty}\left(\mathbb{R}_{+}\right)$.

\footnotetext{
${ }^{2}$ The following pairs of boundary vertices are considered neighbours: $v_{1} \sim v_{2}, v_{2} \sim v_{3}, v_{3} \sim v_{4}$, and $v_{4} \sim v_{1}$.
} 
Consider the solution to this problem in the case where just one control function, say $f_{1}$, is different from zero. Then for sufficiently small values of $t$ the boundary control will generate a d'Alembert wave on the edge $\left[x_{1}, x_{2}\right]$ moving toward the central vertex. This wave will arrive at the central vertex at the moment $t \geq$ $l_{1}=x_{2}-x_{1}$ and will be scattered by it. Since the corresponding vertex scattering matrix is independent of the energy and the reflection coefficient and transition coefficient to the opposite edge are zero (see properties 1-3 formulated at the end of Section 2), the initial d'Alembert wave on the edge $\left[x_{1}, x_{2}\right]$ will generate two scattered d'Alembert waves on the edges $\left[x_{3}, x_{4}\right]$ and $\left[x_{7}, x_{8}\right]$ moving away from the central vertex. These waves have the same profiles as the initial d'Alembert wave multiplied by the scattering coefficients $\alpha$ and $\beta$. Observers placed at the boundary vertices will be able to measure just these scattering coefficients as well as the time-delays $l_{1}+l_{2}$ and $l_{1}+l_{4}$ respectively. Sending waves along all other boundary edges will in addition determine the sign $\sigma$ and the distances $l_{2}+l_{3}$ and $l_{3}+l_{4}$. Hence the boundary observations enable one to reconstruct the matrix $S$ and the distances between any two neighbouring boundary vertices, but not the actual lengths of the edges. It is clear that the described phenomenon is possible only due to very special properties of the vertex scattering matrix $S$.

Let us consider now the scattering problem associated with the quantum cross $\Gamma$. We extend the graph $\Gamma$ by attaching semi-infinite wires $\left(-\infty, x_{2 j-1}\right], j=1,2,3,4$ to all four boundary vertices. Then the scattered waves $\psi$ are solutions to the eigenfunction differential equation (3.1) on the intervals $\Delta_{j}=\left(-\infty, x_{2 j}\right], j=$ $1,2,3,4$ and satisfying the matching conditions (2.2) at the central vertex. Every solution to the differential equation may be written as

$$
\psi(x)=a_{j} e^{-i k\left(x-x_{2 j-1}\right)}+b_{j} e^{i k\left(x-x_{2 j-1}\right)}, x \in \Delta_{j}=\left(-\infty, x_{2 j}\right], j=1,2,3,4 .
$$

The incoming and outgoing amplitudes $b_{j}, a_{j}$ should be chosen so that the function satisfies the matching conditions. Then the scattering matrix $S_{\Gamma}(k)$ is the $4 \times 4$ unitary matrix connecting the vectors of amplitudes

$$
\vec{a}=S_{\Gamma}(k) \vec{b}
$$

Straightforward calculations yield

$$
\begin{aligned}
S_{\Gamma}(k) & =\operatorname{diag}\left(e^{i k l_{1}}, e^{i k l_{2}}, e^{i k l_{3}}, e^{i k l_{4}}\right) S \operatorname{diag}\left(e^{i k l_{1}}, e^{i k l_{2}}, e^{i k l_{3}}, e^{i k l_{4}}\right) \\
& =\left(\begin{array}{cccc}
0 & \alpha e^{i k\left(l_{1}+l_{2}\right)} & 0 & \beta e^{i k\left(l_{1}+l_{4}\right)} \\
\alpha e^{i k\left(l_{1}+l_{2}\right)} & 0 & \sigma \beta e^{i k\left(l_{2}+l_{3}\right)} & 0 \\
0 & \sigma \beta e^{i k\left(l_{2}+l_{3}\right)} & 0 & -\sigma \alpha e^{i k\left(l_{3}+l_{4}\right)} \\
\beta e^{i k\left(l_{1}+l_{4}\right)} & 0 & -\sigma \alpha e^{i k\left(l_{3}+l_{4}\right)} & 0
\end{array}\right) .
\end{aligned}
$$

The matrix $S$ from the matching conditions (2.2) can be recovered: $S=S_{\Gamma}(0)$. The scattering matrix $S_{\Gamma}(k)$ depends on the distances between the neighbouring boundary points in $\Gamma$. The TW-function is a rational transformation of $S_{\Gamma}$,

$$
M(\lambda)=i k \frac{I-S_{\Gamma}(k)}{I+S_{\Gamma}(k)},
$$

and therefore possesses the same property. We proved directly that the crosses with equal distances between neighbouring boundary vertices have identical scattering matrices. 
The infinite crosses just considered are in fact identical, but parameterized in different ways, and therefore the scattering matrices are similar to each other. One may think that this is the reason for the observed phenomenon. Let us consider a slightly more sophisticated example of a double quantum cross formed by two identical crosses (see Figure 2).

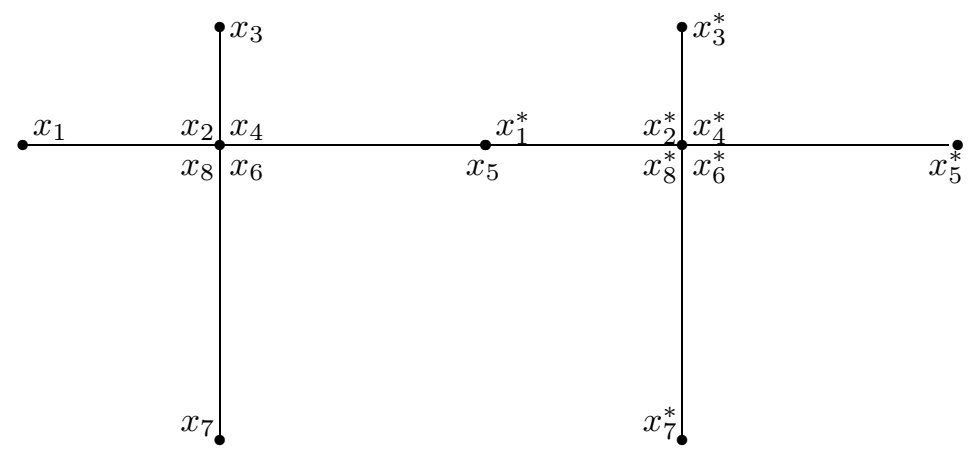

Figure 2. Graph $\Gamma_{2}$. Double quantum cross.

We assume that the lengths are pairwise equal: $l_{j}^{*}=x_{2 j}^{*}-x_{2 j-1}^{*}=x_{2 j}-x_{2 j-1}=$ $l_{j}, j=1,2,3,4$. Consider the Laplace operator $-\frac{d^{2}}{d x^{2}}$ defined on the functions satisfying matching conditions (2.2) at the internal vertices $\left\{x_{2}, x_{4}, x_{6}, x_{8}\right\}$ and $\left\{x^{*} 2, x_{4}^{*}, x_{6}^{*}, x_{8}^{*}\right\}$, standard matching conditions at the removable vertex $\left\{x_{5}, x_{1}^{*}\right\}$ and Dirichlet boundary conditions at the boundary vertices $v_{1}=\left\{x_{1}\right\}, v_{2}=\left\{x_{3}\right\}$, $v_{3}=\left\{x_{3}^{*}\right\}, v_{4}=\left\{x_{5}^{*}\right\}, v_{5}=\left\{x_{7}^{*}\right\}, v_{6}=\left\{x_{7}\right\}$. The scattering matrix for the noncompact graph obtained from $\Gamma_{2}$ by attaching semi-infinite wires to the boundary vertices $v_{j}, j=1,2, \ldots, 6$ can easily be calculated:

$$
S_{\Gamma_{2}}(k)=\left(\begin{array}{cccccc}
0 & \alpha e_{12} & 0 & 0 & 0 & \beta e_{14} \\
\alpha e_{12} & 0 & \sigma \alpha \beta e_{12} e_{23} & 0 & 0 & 0 \\
0 & \sigma \alpha \beta e_{12} e_{23} & 0 & \sigma \beta e_{23} & 0 & 0 \\
0 & 0 & \sigma \beta e_{23} & 0 & -\sigma \alpha e_{34} & 0 \\
0 & 0 & 0 & -\sigma \alpha e_{34} & 0 & -\sigma \alpha \beta e_{14} e_{34} \\
\beta e_{14} & 0 & 0 & 0 & -\sigma \alpha \beta e_{14} e_{34} & 0
\end{array}\right),
$$

where $e_{i j}=e^{i k\left(l_{i}+l_{j}\right)}$. The scattering matrix depends just on the distances between neighbouring boundary vertices in the original cross $\Gamma$, i.e. on the lengths: $l_{1}+$ $l_{2}, l_{2}+l_{3}, l_{3}+l_{4}$, and $l_{4}+l_{1}$. The same transformation as before preserves the scattering matrix, but not the distance $l_{3}+l_{1}$ between the nontrivial internal vertices in $\Gamma_{2}$.

One may generalize the considered counterexamples even for Schrödinger operators with nonzero potentials. Consider any real square integrable potential $q$ on the 
graph $\Gamma$, identically equal to zero on the intervals $\left[x_{4}-l, x_{4}\right]$ and $\left[x_{8}-l, x_{8}\right]$. Consider also the cross graph $\Gamma^{\prime}$ formed by the edges $\left[x_{1}, x_{2}+l\right],\left[x_{3}, x_{4}-l\right],\left[x_{5}, x_{6}+l\right]$ and $\left[x_{7}, x_{8}-l\right]$. The potential $q^{\prime} \in L_{2}\left(\Gamma^{\prime}\right)$ is obtained by extending $q$ by zero to the intervals $\left[x_{2}, x_{2}+l\right]$ and $\left[x_{3}, x_{3}+l\right]$. Let $L_{\sigma, \alpha}(\Gamma)$ and $L_{\sigma, \alpha}\left(\Gamma^{\prime}\right)$ be the corresponding Laplace operators in $L_{2}(\Gamma)$ and $L_{2}\left(\Gamma^{\prime}\right)$ respectively. We assume that the matching conditions (2.2) at the central vertex are satisfied, subject to obvious amendments in the case of $\Gamma^{\prime}$. The same arguments as before can be used to prove that the TW-functions for the Schrödinger operators $L_{\sigma, \alpha}(\Gamma)+q$ and $L_{\sigma, \alpha}\left(\Gamma^{\prime}\right)+q^{\prime}$ are identical.

The results of our studies can be summarized as follows:

Theorem 4.1. The Titchmarsh-Weyl matrix function for a quantum tree (Schrödinger operator on a metric tree) does not necessarily determine the metric tree uniquely.

\section{Conclusions}

The described phenomenon appears to be "rare", and we believe that almost all quantum trees are uniquely determined by their TW-functions. Certain sufficient conditions for trees to be reconstructable are known, but one needs a complete characterization of all such graphs as well as effective numerical algorithms.

Constructed quantum crosses form infinite families, which can be parameterized by one real parameter, and any two members can be continuously deformed into each other preserving the spectrum and the scattering matrix. Note that the scattering matrices not only share the same poles but are just equal. These properties distinguish our example from all known earlier examples.

The spectrum of quantum graphs and the corresponding scattering matrices may be calculated using trace formulas [14, 19] connecting the spectrum to the set of periodic orbits on metric graphs. In the considered example the vertex scattering matrix has zero entries, and therefore some of the periodic orbits do not contribute to the trace formula. Hence the spectrum determines just a subset of periodic orbits leading to ambiguities in reconstructing the metric graph.

Summing up, we conclude that even the simplest quantum trees not always can be reconstructed using boundary measurements.

\section{ACKNOWLEDGEMENT}

The author is grateful to A. Luger for supporting discussions and suggested improvements.

\section{REFERENCES}

1. S. Avdonin and P. Kurasov, Inverse problems for quantum trees, Inverse Probl. Imaging, 2 (2008), no. 1, 1-21. MR2375320 (2008m:35362)

2. S. Avdonin, P. Kurasov, and M. Nowaczyk, Inverse problems for quantum trees. II: Recovering matching conditions for star graphs, Inverse Probl. Imaging 4 (2010), no. 4, 579-598. MR 2726415

3. R. Band, O. Parzanchevski, and G. Ben-Shach, The isospectral fruits of representation theory: quantum graphs and drums, J. Phys. A: Math. Theor., 42 (2009), 175202, 42 pp. MR 2539297

4. R. Band, A. Sawicki, and U.Smilansky, Scattering from isospectral quantum graphs, J. Phys. A 43 (2010), no. 41, 415201, 17 pp. MR 2726689

5. M.I. Belishev, Boundary spectral inverse problem on a class of graphs (trees) by the BC method, Inverse Problems, 20 (2004), 647-672. MR2067494(2005c:34052) 
6. M.I. Belishev and A.F. Vakulenko, Inverse problems on graphs: Recovering the tree of strings by the BC-method, J. Inv. Ill-Posed Problems, 14 (2006), 29-46. MR 2218385

7. J. Boman and P. Kurasov, Symmetries of quantum graphs and the inverse scattering problem, Adv. in Appl. Math., 35 (2005), no. 1, 58-70. MR2141505 (2006g:81051)

8. B.M. Brown and R. Weikard, A Borg-Levinson theorem for trees, Proc. R. Soc. Lond. Ser. A Math. Phys. Eng. Sci., 461 (2005), no. 2062, 3231-3243. MR2172226 (2006d:34061)

9. B.M. Brown and R. Weikard, On inverse problems for finite trees, in "Methods of Spectral Analysis in Mathematical Physics, Conference on Operator Theory, Analysis and Mathematical Physics (OTAMP) 2006", Oper. Theory Adv. Appl., 186, Birkhäuser Verlag, Basel, 2009, 31-48. MR 2732071

10. R. Carlson, Inverse eigenvalue problems on directed graphs, Trans. Amer. Math. Soc., 351 (1999), 4069-4088. MR1473434 (99m:34189)

11. G. Freiling and V.Yurko, Inverse problems for differential operators on trees with general matching conditions, Applicable Analysis, 86 (2007), no. 6, 653-667. MR2345888 (2008g:34016)

12. N.I. Gerasimenko and B. Pavlov, Scattering problems on noncompact graphs, Teoret. Mat. Fiz., 74 (1988), 345-359 (Eng. transl., Theoret. and Math. Phys., 74 (1988), 230-240). MR953298 (90f:47010)

13. N.I. Gerasimenko, Inverse scattering problem on a noncompact graph, Teoret. Mat. Fiz., $\mathbf{7 5}$ (1988), 187-200 (Eng. transl., Theoret. and Math. Phys., 75 (1988), 460-470). MR959124 (89f:81239)

14. B. Gutkin and U.Smilansky, Can one hear the shape of a graph?, J. Phys. A, 34 (2001), 6061-6068. MR1862642 (2002k:05205)

15. M. Harmer, Inverse scattering on matrices with boundary conditions, J. Phys. A, 38 (2005), no. 22, 4875-4885. MR.2148630 (2006b:81349)

16. V. Kostrykin and R.Schrader, Kirchhoff's rule for quantum wires. II. The inverse problem with possible applications to quantum computers, Fortschr. Phys., 48 (2000), no. 8, 703-716. MR 1778728 (2001g:81273)

17. P. Kurasov Inverse problems for Aharonov-Bohm rings, Math. Proc. Cambridge Philos. Soc., 148 (2010), 331-362. MR2600145

18. P. Kurasov On the inverse problem for quantum graphs with one cycle, Acta Physica Polonica A, 116 (2009), 765-771.

19. P. Kurasov and M. Nowaczyk, Geometric properties of quantum graphs and vertex scattering matrices, Opuscula Math., 30 (2010), 295-309. MR2669120

20. P. Kurasov and F. Stenberg, On the inverse scattering problem on branching graphs, J. Phys. A, 35 (2002), 101-121. MR.1891815 (2003e:81207)

21. B.M. Levitan and M.G. Gasymov, Sturm-Liouville differential operators with discrete spectrum, Mat. Sb. (N.S.) 63(105), 445-458 (1964). MR0160962 (28:4171)

22. O. Parzanchevski and R. Band, Linear representations and isospectrality with boundary conditions, J. Geom. Anal., 20 (2010), 439-471. MR2579517

23. T. Shapira and U.Smilansky, Quantum graphs which sound the same, in: Non-Linear Dynamics and Fundamental Interactions NATO Science Series, 2006, 213, Part 1, 17-29.

24. V. Yurko, Inverse spectral problems for Sturm-Liouville operators on graphs, Inverse Problems, 21 (2005), 1075-1086. MR2146822 (2005m:34024)

25. V. Yurko, On the reconstruction of Sturm-Liouville operators on graphs (Russian), Mat. Zametki, 79 (2006), no. 4, 619-630; translation in Math. Notes, 79 (2006), no. 3-4, 572-582. MR:2251311 (2007h:34013)

Department of Mathematics, LTH, Lund University, Box 118, 22100 Lund, Sweden; Department of Mathematics, Stockholm University, 10691 Stockholm, Sweden; and Department of Physics, St. Petersburg University, 198904 St. Peterhof, Russia

E-mail address: kurasov@maths.lth.se, pak@math.su.se 